\title{
MAGNETO-OPTICAL CHARACTERISTICS OF HUMAN SERUM
}

\author{
M. SURMA ${ }^{a}$, J. BuĆKo ${ }^{b}$, G. BĄK ${ }^{c}$ AND M. CISZEK ${ }^{a}$ \\ ${ }^{a}$ Optics Laboratory, Institute of Physics, Adam Mickiewicz University \\ Grunwaldzka 6, 60-780 Poznań, Poland \\ ${ }^{b}$ Institute of Obstetrics and Gynecology, Academy of Medicine \\ Polna 33, 60-535 Poznań, Poland \\ ${ }^{c}$ Greater Poland Cancer Center, Garbary 15, 61-866 Poznań, Poland
}

A study of magneto-optical circular birefringence of human serum from healthy subjects and cancer patients was performed. Significant differences in the magnetic field induced change of the natural optical activity of serum from healthy blood donors and neoplasmic patients were noted. The natural optical activity of blood is positive, irrespective of the donor health status, whereas the magnetic field induced $(B>10 \mathrm{~T})$ change in the natural optical activity of the serum from cancer patients is negative while that from healthy donors is positive. The value and sign of the characteristic magneto-optical marker of human serum was discovered in a study of the influence of very high magnetic fields on chiral media and interpreted on the basis of the recent developments in the theory of magnetic field induced optical circular birefringence in the media revealing natural optical activity. The blood serum is a chiral medium.

PACS numbers: $42.25 . \mathrm{Lc}, 78.20 . \mathrm{Ek}, 78.20 . \mathrm{ss}$

\section{Introduction}

Magneto-optical circular birefringence (MOCB) of an atomic or molecular medium is the effect of rotation of the polarization plane of linearly polarized light induced by magnetic field. A beam of light characterized by the wave vector $k$ is passed through a medium over the length $L$, in a magnetic field of induction $\boldsymbol{B}$, in the Faraday geometric configuration of the vectors: $\boldsymbol{B}\|\boldsymbol{k}\| \boldsymbol{L}$. The appearance of magneto-optical circular birefringence induced in the medium by a magnetic field $\boldsymbol{B}$ described by the relation: $\alpha(B)=V \boldsymbol{B} \cdot \boldsymbol{L}$, where $\alpha(B)$ is the angle of rotation of the light polarization plane induced by the magnetic field $\boldsymbol{B}$, is known as the linear Faraday effect. The symbol $V$ stands for the Verdet constant characteristic of a given medium dependent on its temperature and wavelength of the incident light. 
Measurements performed in this study have shown that the value of the Verdet constant does not reflect the health status (the occurrence of cancer) of the blood donor. Moreover, measurements performed for different samples (including water) in very strong magnetic fields [1] have not revealed any effect of magnetic field on the values of the Verdet constant. The theory of the Faraday effect predicts a contribution of the nonlinear component induced by the magnetic field and proportional to $B^{3}: \alpha\left(B^{3}\right)=\tilde{V} B^{3} L$, known as the non-linear Faraday effect. This effect has not been observed for the media not showing the natural optical activity (non-chiral media) because their $\tilde{V}$ is too low and a measurement of $\alpha\left(B^{3}\right)$ is beyond current experimental possibilities.

The blood serum is characterized by natural optical activity (NOA), which is a resultant of NOA of all its components, including aminoacids and proteins. The positive value of NOA of blood serum, $\alpha_{0}>0$ (the angle $\alpha_{0}$ of rotation of the polarization plane of the light passing through the medium), is clockwise when looking along the light beam $k$ vector, which means the dominant contribution of dextrorotatory components in the serum. In natural biopolymers such as proteins laevorotatory behavior has not been reported. In chiral pure chemical compounds, e.g. tartaric acid, there are two enantiomers: dextro- $(+)$ and laevorotatory $(-)$, whose natural chirality for $B=0$ is expressed as the angle $\alpha(0) \equiv \alpha_{0}$ of polarization plane rotation of light passing in the medium over a distance $L=1 \mathrm{~mm}$, and equals $+\alpha_{0}$ and $-\alpha_{0}\left[\mathrm{deg} \mathrm{mm}^{-1}\right.$ ], respectively, and the following relation holds: $\left|+\alpha_{0}\right|=\left|-\alpha_{0}\right|[1,2]$. Natural aminoacids such as e.g. leucine and threonine, occurring in proteins, are dextrorotatory $(+)$. As shown in [2] in a strong magnetic field $B$ the natural optical activity of enantiomers $(+)$ increases by a factor depending on $B^{2}$ and equals $\alpha\left(B^{2}\right)=b B^{2} L>0$. For enantiomers $(-)$ the analogous change depending on $B^{2}$ can be written as $\alpha\left(B^{2}\right)=-b B^{2} L$. The sign plus at $+\alpha(0)$ and $+\alpha\left(B^{2}\right)$ means the dextrorotatory change, while the sign minus at $-\alpha(0)$ and $-\alpha\left(B^{2}\right)$ means that the change is laevorotatory. The total rotation of the light polarization plane induced by a strong magnetic field in a chiral medium is a result of three effects: the linear Faraday effect $\alpha(B)=a B L$, nonlinear Faraday effect $\alpha\left(B^{3}\right)=c B^{3} L$, and the effect caused by the change in the natural optical activity of a chiral medium $\alpha\left(B^{2}\right)=b B^{2} L$. Therefore, the total magneto-optical effect of induced circular birefringence $\alpha_{\exp }$ in a chiral medium can be expressed as follows for the two configurations $\boldsymbol{B}\|\boldsymbol{k},-\boldsymbol{B}\| \boldsymbol{k}$ :

$$
\begin{aligned}
& (\boldsymbol{B} \| k) \quad \alpha^{\uparrow \uparrow}=a B L+b B^{2} L+c B^{3} L, \\
& (-\boldsymbol{B} \| k) \quad \alpha^{\uparrow \downarrow}=-a B L+b B^{2} L-c B^{3} L,
\end{aligned}
$$

with the coefficients $a>0, b>0, c>0$. The configurations $(\boldsymbol{B} \| k)$ and $(-\boldsymbol{B} \| k)$ mean that the senses of the vectors $B$ and $k$ are either the same or the opposite. Equations (1) and (2) are the fundamental relations for determination of the value of the coefficient $b$, which can be used as a magneto-optical characteristic marker of the blood serum.

The theory predicting the MOCB dependence on the magnetic field induction in three powers $B, B^{2}, B^{3}$ is given in [3-5] and a correct interpretation of the contribution depending on $B^{2}$, for a chiral medium is presented in [5] and confirmed in $[1,2]$. 
The first measurements of the light polarization plane rotation $\alpha^{\uparrow \uparrow}$ and $\alpha^{\uparrow \downarrow}$ (Eqs. (1), (2)) in which the MOCB terms depending on $B^{2}$ and $B^{3}$ were observed for tartaric acid and blood serum from ovary cancer patients, were performed in 1996 at the Optics Laboratory, Institute of Physics, Adam Mickiewicz University, Poznań, and published in [6].

For the blood serum samples from three patients diagnosed with ovaries cancer, studied in [6], negative values of coefficient $b(b<0)$ were obtained, although their natural optical activity was positive.

The blood serum is not a chemically pure enantiomer. The resultant value of its natural optical activity is positive $\alpha(0)>0$, but the magnetic field induced effect in the samples from cancer patients $\alpha\left(B^{2}\right)$ can be negative. The results $\alpha_{0}>0$ and $\alpha\left(B^{2}\right)<0$ do not correspond to the signs: $\alpha_{0}>0$ and $\alpha\left(B^{2}\right)>0$, and/or $\alpha_{0}<0, \alpha\left(B^{2}\right)<0$ which hold for chemically pure enantiomers [1]. The disagreement between the sign of NOA and that of $b$ coefficient of the blood serum from cancer patients $\left(\alpha_{0}>0, b<0\right)$ has prompted comparative studies on magneto-optical circular birefringence in blood serum samples from (i) cancer patients, (ii) cancer patients in remission or at different stages of therapy, (iii) healthy subjects.

To this aim, in cooperation with the Greater Poland Cancer Center and the Institute of Obstetrics and Gynecology at the Academy of Medicine in Poznan, analyses of over 100 samples of blood serum from cancer patients were made. For the sake of comparison MOCB was also studied in blood serum of over 20 healthy subjects.

\section{Experimental}

The unique equipment for MOCB measurements is described in [6]. The same paper gives a method of analysis of the observed MOCB effect in which the contribution due to the Faraday effects (linear $\alpha(\boldsymbol{B})=a B L$ and non-linear $\left.\alpha\left(B^{3}\right)=c B^{3} L\right)$ the effect proportional to $B^{2}$, a change in the natural optical activity of blood serum $\alpha\left(B^{2}\right)=b B^{2} L$. The method of separating the contributions proportional to $B, B^{2}$, and $B^{3}$ have been derived in $[1,2]$ and used for analysis of experimental results in the present work.

Equations (1) and (2) imply that the best method of measurement of the coefficient $b$ involves: (1) measurement of the angle $\alpha^{\Uparrow \uparrow}$, then change of the magnetic field sense from $+\boldsymbol{B}$ to $-\boldsymbol{B}$, with respect to the sense of the light propagation vector $k$ and (2) measurement of the angle $\alpha \uparrow$. The sense of the vector of the magnetic field induction from $+\boldsymbol{B}$ to $-\boldsymbol{B}$, is realized by a commutation of the direction of the current flow in the coil producing strong magnetic field. If the serum studied is characterized by the positive values of the coefficients $a>0, b>0, c>0$, then the measured values $\alpha^{\uparrow \uparrow}$ and $\alpha^{\uparrow \downarrow}$ are defined by Eqs. (1) and (2), and the sum

$$
\alpha^{\uparrow \uparrow}+\alpha^{\uparrow \downarrow}=2 b B^{2} L
$$

and

$$
b=\left(\alpha^{\uparrow \uparrow}+\alpha^{\uparrow \downarrow}\right) / 2 B^{2} L
$$


If the serum is characterized by the positive values of the coefficients $a$ and $c$ $(a>0, c>0)$ but a negative value of $b(b<0)$, then on the basis of Eqs. (1) and (2) we obtain

$$
\begin{aligned}
& (\boldsymbol{B} \| k) \quad \alpha^{\uparrow \uparrow}=a B L-b B^{2} L+c B^{3} L, \\
& (-\boldsymbol{B} \| k) \quad \alpha^{\uparrow \downarrow}=-a B L-b B^{2} L-c B^{3} L,
\end{aligned}
$$

and

$$
\alpha^{\uparrow \uparrow}+\alpha^{\uparrow \downarrow}=-2 b B^{2} L
$$

and from Eq. (7) we have

$$
b=-\left(\alpha^{\Uparrow \uparrow}+\alpha^{\dagger \downarrow}\right) / 2 B^{2} L,
$$

where $\alpha^{\uparrow \uparrow}$ and $\alpha^{\uparrow \downarrow}$ (Eqs. (4) and (8)) are the experimentally determined angles of rotation: $\alpha^{\uparrow \uparrow} \equiv \alpha_{\exp },(\boldsymbol{B} \| k)$ - parallel configuration of the vectors $\boldsymbol{B}$ and $\boldsymbol{k}$ and $\alpha^{\uparrow \downarrow} \equiv \alpha_{\text {exp }},(-\boldsymbol{B} \| k)$ - antiparallel configuration of the vectors $\boldsymbol{B}$ and $k$. In the experimental practice the sense of the rotation $\alpha \uparrow \uparrow$ is positive (dextrorotatory rotation) and the sense of $\alpha \uparrow$ is negative (laevorotatory rotation), because in MOCB the linear Faraday effect dominates, and is of several orders of magnitude greater than the contribution of $\alpha\left(B^{2}\right)$ and $\alpha\left(B^{3}\right)$.

For blood serum made in $90 \%$ of water, also the linear Faraday effect $\alpha(B)$ dominates in MOCB and is a few orders of magnitude greater than the effects $\alpha\left(B^{2}\right)$ and $\alpha\left(B^{3}\right)$. Thus, the effective rotation angle $\alpha^{\uparrow \uparrow}$ (configuration $\boldsymbol{B} \| \boldsymbol{L}$ ) gives the clockwise rotation implied by the Faraday effect for $a>0$ (Verdet constant $V>0$ ) while the rotation $\alpha^{\uparrow \downarrow}$ is counterclockwise. In weak magnetic fields ( $B \approx 1 \mathrm{~T}$ ) the effects $\alpha\left(B^{2}\right)$ and $\alpha\left(B^{3}\right)$ are not measurable but the linear Faraday effect can be measured

$$
\alpha(B)=\left|\alpha^{\uparrow \uparrow}(B)\right|=\left|\alpha^{\uparrow \downarrow}(B)\right|=a B L .
$$

In a very strong magnetic field $(B>15 \mathrm{~T})$ by the method of fitting $\alpha^{\uparrow \uparrow}$ and $\alpha^{\uparrow \downarrow}$ we obtain the value of the coefficient $c$. The relations $\alpha_{\text {fit }}^{\uparrow \uparrow}=\left(a B+b B^{2}+c B^{3}\right) L$ and $\alpha_{\text {fit }}^{\uparrow \downarrow}=\left(-a B+b B^{2}-c B^{3}\right) L$ satisfy the experimentally measured relations $\alpha^{\uparrow \downarrow}, \alpha^{\uparrow \downarrow}$. The error in the measurement of the coefficients $a$ and $b$ does not exceed $\pm 4 \%$, while that of determination of $c$ does not exceed $\pm 10 \%$.

The natural optical activity of blood serum is positive $\left(\alpha_{0}>0\right)$, and the linear Faraday effect is positive $a>0$, whereas the value and sign of the coefficients $b$ and $c$ establish the magneto-optical characteristics of human serum.

\section{Results}

\subsection{Magneto-optical characteristics of the serum donors}

Blood serum samples from healthy blood donors were obtained from the Blood Service Laboratory and subjected to MOCB studies after centrifugation and removal of fibrinogen. From among 24 samples of serum studied, Table I presents results for 10 samples for which the following parameters were determined: magneto-optical coefficients $a, b$, and $c$, group of blood and $\mathrm{Rh}$, natural 
optical activity $\alpha_{0}$, the parameter $\alpha_{0} / b$ characterizing the relation between the natural optical activity $\alpha_{0}$ and its change induced by a strong magnetic field $\alpha\left(B^{2}\right)=b B^{2} L$.

In Fig. 1 the experimental results of $\alpha^{\uparrow \uparrow}$ and $\alpha^{\uparrow \downarrow}$ for serum 69 (No. 10, Table I) are presented.

TABLE I

Natural optical activity $\alpha_{0}$, magneto-optical coefficients $a, b$ and $c$, blood group, Rh and the value of $\alpha_{0} / b$ for selected 10 samples of healthy blood donors.

\begin{tabular}{c|c|c|c|c|c|c}
\hline \hline No. & $\begin{array}{c}\alpha_{0} \times 10^{3} \\
{\left[\frac{\mathrm{deg}}{\mathrm{mm}}\right]}\end{array}$ & $\begin{array}{c}a \times 10^{3} \\
{\left[\frac{\mathrm{deg}}{\mathrm{T} \mathrm{mm}}\right]}\end{array}$ & $\begin{array}{c}b \times 10^{5} \\
{\left[\frac{\mathrm{deg}}{\mathrm{T}^{2} \mathrm{~mm}}\right]}\end{array}$ & $\begin{array}{c}c \times 10^{8} \\
{\left[\frac{\mathrm{deg}}{\mathrm{T}^{3} \mathrm{~mm}}\right]}\end{array}$ & $\begin{array}{c}\left(\alpha_{0} / b\right) \times 10^{-2} \\
{\left[\mathrm{~T}^{2}\right]}\end{array}$ & Blood group/Rh \\
\hline 1 & 56 & 332 & 1.6 & 2.62 & 35.00 & $0 /+$ \\
2 & 62 & 324 & 2.0 & 1.60 & 31.00 & $\mathrm{~A} /-$ \\
3 & 64 & 336 & 2.4 & 0.34 & 26.60 & $\mathrm{~B} /-$ \\
4 & 66 & 328 & 5.0 & 3.04 & 13.20 & $\mathrm{~A} 1 /+$ \\
5 & 58 & 338 & 6.4 & 0.76 & 9.06 & $0 /+$ \\
6 & 75 & 320 & 16.4 & 1.74 & 4.57 & $\mathrm{~B} /+$ \\
7 & 57 & 334 & 19.0 & 0.58 & 3.00 & $\mathrm{~A} 1 /+$ \\
8 & 62 & 326 & 20.0 & 1.20 & 3.10 & $0 /-$ \\
9 & 64 & 328 & 38.0 & 1.32 & 1.68 & $0 /-$ \\
10 & 49 & 328 & 30.0 & 2.00 & 1.63 & $\mathrm{AB} /-$
\end{tabular}

$\alpha(\operatorname{deg})$

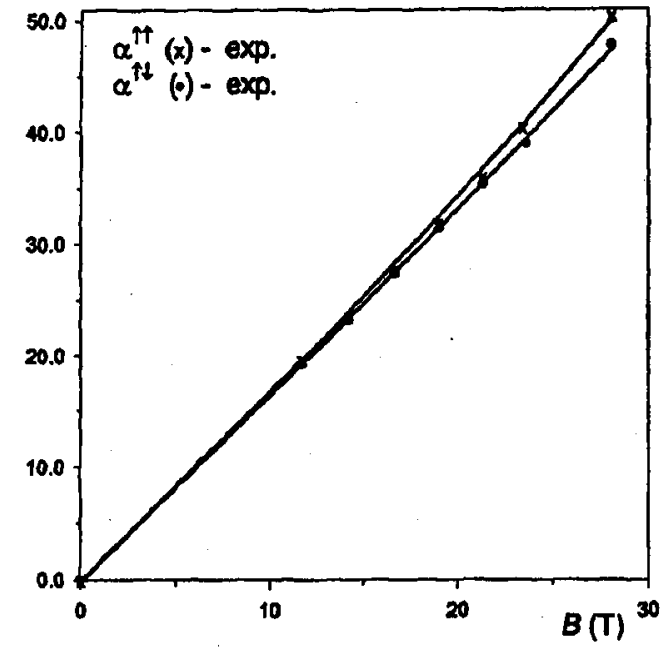

Fig. 1. Magneto-optical circural birefringence $\alpha^{\dagger \dagger}(*)$ and $\alpha^{\dagger \downarrow}(\bullet)$ of serum 69 from healthy donor: $b>0$. 
The measurements were performed for the light wavelength $(\lambda=488 \mathrm{~nm})$ in the sample of the length $L_{\exp }=5 \mathrm{~mm}$. Therefore, the values of $\alpha_{0}$ and $a, b$, and $c$ coefficients are in $\operatorname{deg} \mathrm{mm}^{-1}, \operatorname{deg} \mathrm{T}^{-1} \mathrm{~mm}^{-1}, \operatorname{deg} \mathrm{T}^{-2} \mathrm{~mm}^{-1}$ and $\operatorname{deg} \mathrm{T}^{-3} \mathrm{~mm}^{-1}$, respectively.

\subsection{MOCB characteristics of blood serum from cancer patients}

The first results of MOCB for blood serum samples recorded among others for three women suffering from ovary cancer, were obtained in 1997 [6]. The samples of blood serum from cancer patients are characterized by a negative value of the magneto-optical coefficient $b(b<0)$. For the samples from patients with advanced cancer also the magneto-optical coefficient $c$ is negative $(c<0)$. Exemplary serum 94, rectal cancer (Table II, No. $2-11.06 .97$ ) the results of $\alpha^{\uparrow \uparrow}$ and $\alpha^{\uparrow \downarrow}$ for $L=5 \mathrm{~mm}$ are presented (Fig. 2).

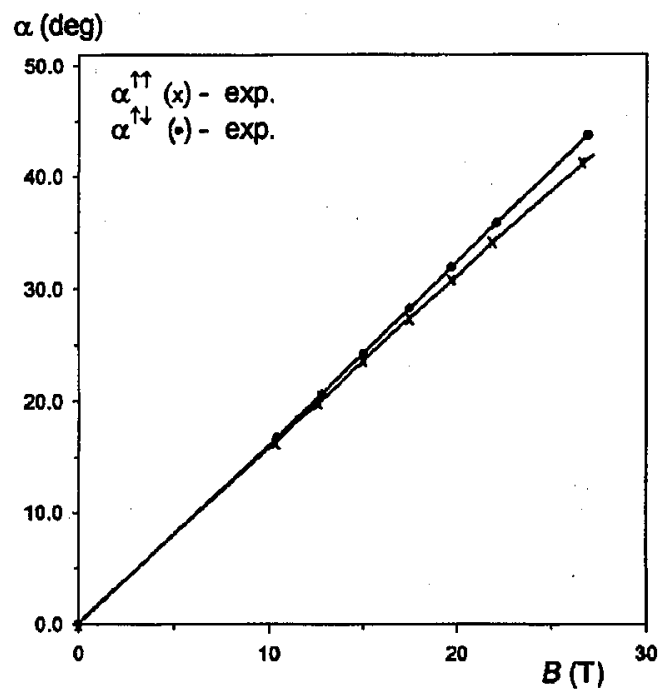

Fig. 2. Magneto-optical circural birefringence $\alpha^{\dagger \dagger}(*)$ and $\alpha^{\dagger \downarrow}(\bullet)$ of serum 94 from rectal cancer patient: $b<0$.

The X-ray diffraction pattern of the samples characterized with $(b<0$, $c<0$ ) reveal a disappearance of the $I_{\mathrm{eu}} / N$ local maximum characteristic of diffraction in pure water where $I_{\mathrm{eu}}$ is the intensity of X-ray diffracted radiation, expressed in electron units per the number $N$ of diffraction centers $[7,8]$. The disappearance of the local maximum $I_{\text {eu }} / N$ for the samples of blood serum from cancer patients is explained by destruction of the tetrahedral structure of water as a result of the presence of neoplasmic mutants of proteins [8]. The evidence of destruction of the tetrahedral structure of water was also observed to be caused by the presence of salts in high concentrations [9]. In the blood serum samples from healthy people, the tetrahedral structure of water is preserved [8] as it is in solutions of low concentration of salts [9]. 


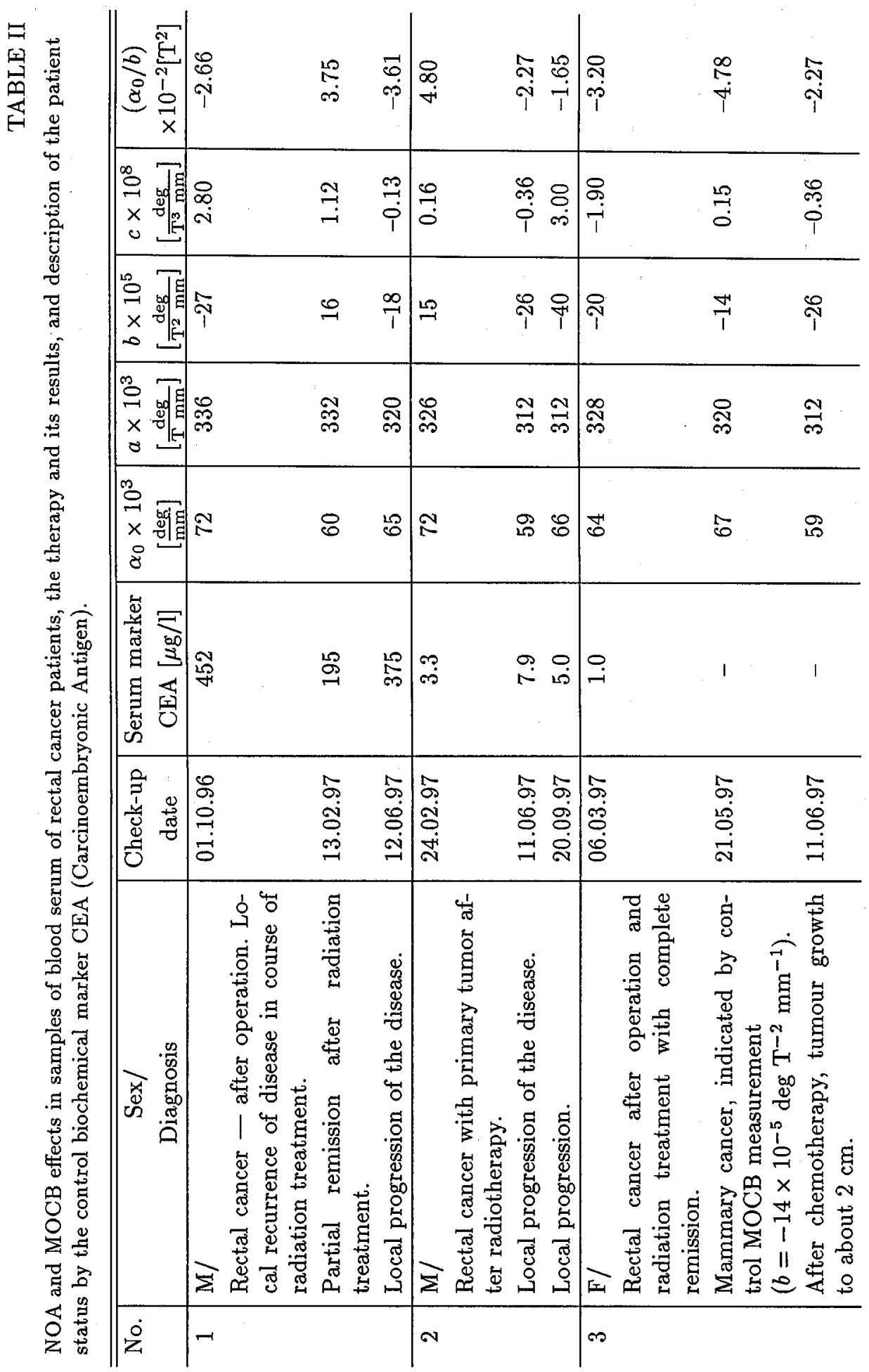


To illustrate the possibilities of MOCB measurements for recognition and analysis of the health status of cancer patients, Table II gives the magneto-optical characteristics of blood serum samples for three patients $(M-$ males and $F-f e-$ males) with rectal cancer. Relations between the biochemical CEA markers and the values of $b$ coefficient are fully consistent and in a $100 \%$ correlation. The changes in values of the magneto-optical coefficient $c$ do not correlate with the changes in CEA marker and $b$ recorded in the period from 01.10 .96 to 12.06 .97 and the change from

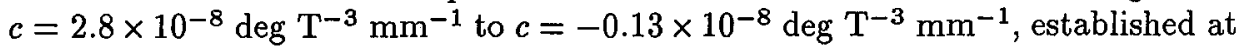
different check-ups. The data presented in Table II for the three patients have been chosen to illustrate the usefulness of the MOCB method for clinical diagnostics and are selected from the material for 20 rectal cancer patients. The results for all 20 patients are fully consistent with the examples given in Table II. According to the estimations of MOCB sensitivity; a number of $N \approx 10^{14}$ of laevorotatory biomolecules is detectable by the MOCB method. In blood serum the dextrorotatory biomolecules dominate and therefore, the total NOA is positive $\left(\alpha_{0}>0\right)$ and the contribution $(B=0)$ of laevorotatory mutants is undetectable. The correlation of the values of magneto-optical coefficient $b$ obtained by the MOCB method, applied for the first time in [6], with the health status of chosen patients makes the basis for assuming the value of this parameter as indicating (i) the presence of cancer, (ii) the effects of therapy and (iii) changes at subsequent stages of therapy.

\section{Discussion}

The analysis of the magneto-optical characteristics of blood serum from cancer patients and healthy people suggests the occurrence of specific biopolymers in the serum samples from the former group. These are most probably chiral mutants characterized by the magnetic field induced laevorotatory circular birefringence, contrary to the dextrorotatory biopolymers in the serum samples of healthy subjects.

In healthy organisms the occurrence of laevorotatory structures is not observed and when artificially introduced, they are destroyed. Therefore, the magneto-optical coefficient $b$ of blood serum brings the information of the presence or absence of a disease.

The theory describing among others nonlinear effects related to the influence of magnetic field on a chiral medium and proportional to $B^{3}$ [3] suggests that $c<0$ signalizes the presence of free radicals in blood serum of cancer patients. This conclusion is supported with results of the studies proving the role of free radicals in generation of cancer, e.g. mammary cancer [10]. Moreover, it can also indicate that the MOCB method may be sensitive for detection of free radicals.

Natural optical activity is positive for all samples of blood serum studied $\left(\alpha_{0}>0\right)$. The result $\alpha_{0}>0$ simply means that dextrorotatory molecules dominate in a given serum sample. The values $a>0$ testify to the dominant probability of the dipolar transitions of molecules characterized by a positive value of the Verdet constant $[1,5]$. Since the main component of blood serum is water for which the Verdet constant is $a=V=0.314 \operatorname{deg} \mathrm{T}^{-1} \mathrm{~mm}^{-1}, \lambda=488 \mathrm{~nm}$, for

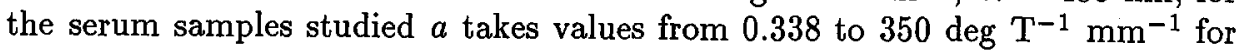




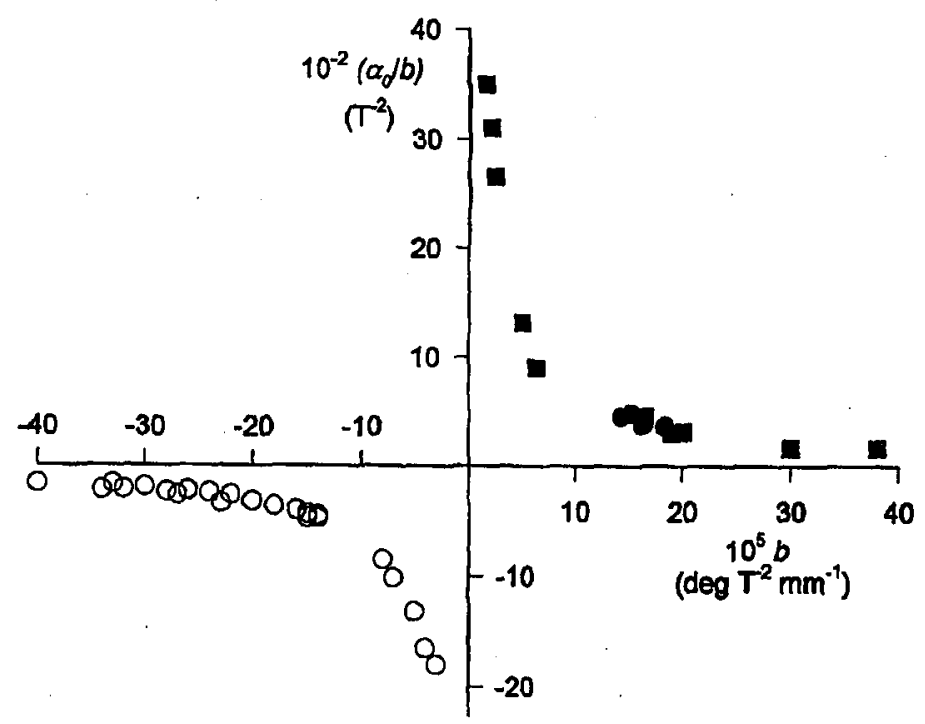

Fig. 3. Numerical relation between the natural optical activity $\alpha_{0}$ and $B^{2}$ induced circular birefringence parameter $b, \alpha\left(B^{2}\right)=b B^{2} L$, of serum: healthy donors, $b>0$ (a); recovered patients, $b>0(\bullet)$; and cancer patients; $b<0(0)$.

healthy subjects and from 0.300 to $0.352 \mathrm{deg} \mathrm{T}^{-1} \mathrm{~mm}^{-1}$ for cancer patients. The distribution of the values of $a$ does not correspond to the changes in $b$ and $c$. The value of $\alpha_{0} / b$ is of the order of $10^{2}$, in $\mathrm{T}^{2}$ and gives the information on the change in the natural optical activity induced by the magnetic field, relative to $\alpha_{0}$ (Fig. 3). As follows from these data the induced effect proportional to $B^{2}$ is three to five orders of magnitude lower than NOA of serum samples. Water being non-chiral medium does not contribute to the value of $\alpha\left(B^{2}\right)$. However, as indicated by $\mathrm{X}$-ray diffraction studies [8], changes in the structure of biomolecules caused by the presence of cancer in the organism $(b<0)$ lead to a destruction of the tetrahedral structure of water in blood serum. Magneto-optical characteristics of blood serum is reflected in the value of the coefficient $b$ (marker $b$ ). Experimental values of this coefficient (Fig. 3) measured for blood serum of healthy and cancer subjects allow drawing the following conclusions:

1) For blood serum of healthy subjects: $b>0, \alpha_{0}>0$.

2) For blood serum of cancer patients with deteriorating health status: $b<0$, and decreases with increasing deterioration, the value of $\alpha_{0}$ is lower than the mean obtained for stable conditions.

3) For blood serum of cancer patients of stable health status: $b<0$, $\alpha_{0} \approx$ const.

4) For blood serum of cancer patients with improving health status: $b \leq 0$.

\section{Final remarks}

Magneto-optical study of blood serum in strong magnetic fields of induction over $10 \mathrm{~T}$ revealed that the magneto-optical characteristics can be a source of 
information about the presence of cancer in a given organism. An unambiguous and direct relationship was found between the sign and value of the coefficient $b$ $(b<0)$ for blood serum samples of cancer patients.

At the present stage of statistical analysis, the method cannot provide decisive information on the type of cancer. It is expected however that future studies will enable collecting enough statistical data to offer a possibility of such conclusions. The effects proportional to $B^{2}$ and $B^{3}$ in MOCB were discovered in 1997. In the same year the first MOCB measurements of blood serum samples were made [6]. The method has been so far presented at the international conference of the European Physical Society in Grenoble in 1998 [11] and in London in 1999 [7].

Encouraged by the promising results of the MOCB method, we believe that it is purposeful to continue the studies.

The amount of blood serum needed for the MOCB test is not greater than $0.5 \mathrm{ml}$, while the sensitivity of recording the effect proportional to the square induction of the magnetic field $\alpha\left(B^{2}\right)=b B^{2} L$ and $b$ value measurement is determined by the threshold density of $10^{14}$ dextrorotatory and/or laevorotatory carriers in blood serum in a magnetic field of $B \approx 20 \mathrm{~T}$ and the optical field of laser light produced by a laser of the power of the order $50 \mathrm{~mW}$. The aim of this paper is to present the, so far unknown, magneto-optical characteristic of human serum and to arouse interest in the possible usefulness of MOCB method for cancer diagnosis and control.

\section{References}

[1] M. Surma, Mol. Phys. 93, 271 (1998).

[2] M. Surma, Mol. Phys. 96, 429 (1999).

[3] C. Graham, R.E. Raab, Proc. Phys. Soc. 90, 417 (1967).

[4] P.W. Atkins, M.H. Miller, Mol. Phys. 15, 491 (1968).

[5] R. Zawodny, S. Woźniak, G. Wagnière, Mol. Phys. 91, 165 (1997).

[6] M. Surma, Mol. Phys. 90, 993 (1997).

[7] A. Mikusińska-Planner, M. Surma, in: Proc. 11th General Conf. EPS, London 1999, Abstract Book PI.P4.6, Institute of Physics, London 1999, p. 78.

[8] A. Mikusińska-Planner, M. Surma, Spectrochim. Acta, Part A, Mol. Biomol. Spectrosc. 56, 1835 (2000).

[9] P. Terpstra, D. Combes, A. Zwiek, J. Chem. Phys. 92, 65 (1990).

[10] R.K. Murray, D.K. Granner, P.A. Mayes, V.W. Rodwell, Biochemia Harpera, 3rd ed., Wydawnictwo Lekarskie PZWL, Warszawa 1995, Ch. 89, p. 910 (in Polish).

[11] M. Surma, M. Ciszek, in: Proc. 17th General Conf. EPS, Condensed Matter Division, Grenoble 1998, Book of Abstracts 22E:26.14, Ed. J. Dumas, Grenoble 1998, p. 224. 